\title{
Probing Physics and Chemistry in Circumnuclear Torus with $\mathrm{OH}$
}

\author{
Yu Zhi-yao \\ Shanghai Astronomical Observatory,80 Nandan Road,Shanghai \\ 200030, China \\ National Astronomical Observatories, Beijing 100012, China \\ E-mail: zyyu@center.shao.ac.cn
}

\begin{abstract}
We observed $\mathrm{OH}$ maser emission at $18 \mathrm{~cm}$ in a circumnuclear torus surrounding the center of IRAS10173+0828 using the 7 telescopes of MERLIN, together with the Lovell antenna. IRAS10173+0828 is a distant super-luminous far-infrared galaxy. The $\mathrm{OH}$ maser emission is remarkably narrow (FWHP $=$ $39 \mathrm{~km} \mathrm{~s}^{-1}$ ) for its strength, and the 1667 and $1665 \mathrm{MHz}$ lines are well separated. The 1667 transition shows two distinct peaks displaced from one another by $100 \mathrm{~km} \mathrm{~s}^{-1}$. Using our MERLIN observational results we probe the physics in the circumnuclear torus surrounding the center of IRAS10173+0828, obtain the kinematic properties of the torus, and study the central source.
\end{abstract}

\section{Introduction}

In January 2002 we observed line emission from $\mathrm{OH}$ masers in a region surrounding the center of IRAS10173+0828 using MERLIN. Powerful OH maser emission has been previously detected in the circumnuclear disk of Arp220, IIIZw35, Mrk273, and IRAS17208-0014 by Lonsdale et al. (1998), Diamond et al. (1999), Yates et al.(2000), and Diamond et al.(1999), respectively. Previous VLBI observation and time series analysis of the spectra (Haschick, Bann \& Peng 1994) suggests that the masers originate in a rotating torus surrounding a massive object (with a mass $\mathrm{M}$ of $1.5 \times 10^{7} \mathrm{M}_{\odot}$ ) at the center of the galaxy (Watson \& Wallin 1994). IRAS10173+0828 is a distant super-luminous far-infrared galaxy (Mirabel \& Sanders 1987). The $\mathrm{OH}$ maser emission is remarkably narrow $\left(\mathrm{FWHP}=39 \mathrm{~km} \mathrm{~s}^{-1}\right.$ ) for its strength, and the 1667 and $1665 \mathrm{MHz}$ lines are well separated. The $1667 \mathrm{MHz}$ transition shows two distinct peaks separated by $100 \mathrm{~km} \mathrm{~s}^{-1}$. Using our MERLIN observational results we probe the physics in the circumnuclear torus surrounding the center of IRAS10173+0828, obtain the kinematic properties of the torus, and study the central source.

\section{Observations}

We observed IRAS10173+0828 for $2 \times 12$ hrs on $24-25$ Jan. 2002 using the 7 telescopes of MERLIN, (operated by the University of Manchester on behalf of PPARC), and including the Lovell antenna. The point-like quasar $0552+398$ was used as the bandpass and flux calibration source. IRAS10173+0828 was observed by switching between two $4-\mathrm{MHz}$ bands containing 128 frequency chan- 
nels each. The bands were centered on 1588.5 and $1591.5 \mathrm{MHz}$. The total time on IRAS10173+0828 was $14.78 \mathrm{hr}$. In between each 4 min scan on IRAS10173+0828, the phase reference source $1015+057$ was observed at 1590 $\mathrm{MHz}$ using a normal $16 \mathrm{MHz}$ bandwidth, averaged to a single $14.5 \mathrm{MHz}$ channel for data processing. $0552+398$ was observed at all frequencies and configurations. All further processing was done in AIPS. The phase and amplitude of $0552+398$ were calibrated and the data were used to derive tables of bandpass corrections for the both $4-\mathrm{MHz}$ data sets. These data also showed that there were no instrumental phase changes associated with observing configuration or frequency changes. We self-calibrated the amplitude and phase of $1015+057$ and applied these solutions and the bandpass corrections to the IRAS10173+0828. We then re-weighted the data from each antenna in proportion to its sensitivity. $1015+057$ was observed at $101827.8483,+053029.936(\mathrm{~J} 2000)$ and the pointing position of IRAS10173+0828 was 101959.9 , +08 13 34(J2000). The final absolute position accuracy of the components of IRAS10173 is 20 mas, plus a signal-to-noise-dependent relative error. We plotted the calibrated visibility amplitudes and phases of IRAS10173+0828 as a function of channel. We also converted the frequency axis to velocity. Using a line rest frequency of $1667.359 \mathrm{MHz}$, the velocity in channel 64 of the data set observed at 1588.5 $\mathrm{MHz}$ is $\mathrm{v}_{l s r}=14179.26 \mathrm{~km} \mathrm{~s}^{-1}$. The data centered on $1588.5 \mathrm{MHz}$ line showed a feature at the expected position of the $1667 \mathrm{MHz}$ line but the data centered on $1591.5 \mathrm{MHz}$ had a flat spectrum. We also Fourier transformed these continuum data without cleaning to give a one-channel dirty map, and similarly made a 128-channel dirty map of the data containing the line as well as continuum. We then subtracted the continuum map from each channel of line+continuum data. Finally we cleaned the resulting line-only data-cube, using a 200 mas FWHM circular restoring beam to make the maps easier to interpret visually. Note that the natural beam fitted to unweighted data is $286 \times 171$ mas; we checked that using a circular beam did not produce artifacts. The typical noise in a quite channel is $\sigma_{r m s}=1 \mathrm{mJy}$ beam ${ }^{-1}$, rising to $2 \mathrm{mJy}_{\text {beam }}{ }^{-1}$ in the brightest channel due to dynamic range limitations arising from the sparse coverage of the visibility plane.

\section{Results}

We detected a signal at $>3 \sigma$ in 19 velocity channels, out of 128 channels observed, and obtained the contour maps of $\mathrm{OH}$ maser in the 19 velocity channels. The physical parameters of the $\mathrm{OH}$ maser in the 19 velocity channels are shown in Table 1.

We find that the contour-maps of $\mathrm{OH}$ maser in some velocity channels extend to both east and west. The morphology of the contour is warped. This implies to us that the circumnuclear torus around the center of IRAS10173+0828 is warped. Using Yu's (1996) model we obtain from Table 1 that the mass of central black hole is $3 \times 10^{10} \mathrm{M}_{\odot}$, that is, evidence of a massive black hole.

Acknowledgments. This work was supported by the NATIONAL NATURAL SCIENCES FOUNDATION OF CHINA. MERLIN is a national facility operated by the University of Manchester on behalf of PPARC. I thank Dr. 
Table 1. The physical parameters of $\mathrm{OH}$ maser in the 19 velocity channels*

\begin{tabular}{ccccccc}
\hline CHAN No & $\begin{array}{c}\text { VELOCITY } \\
(\mathrm{km} / \mathrm{s})\end{array}$ & $\begin{array}{c}\text { RA }_{\text {offs }} \\
(\mathrm{mas})\end{array}$ & $\begin{array}{c}\text { error } \\
(\mathrm{mas})\end{array}$ & $\begin{array}{c}\text { Dec }_{\text {offs }} \\
(\mathrm{mas})\end{array}$ & $\begin{array}{c}\text { error } \\
(\mathrm{mas})\end{array}$ & $\begin{array}{c}\text { Peak } \\
(\mathrm{mJy} / \text { beam })\end{array}$ \\
\hline 87 & 14050 & 4468 & 65 & -195 & 67 & 5 \\
88 & 14044 & 4457 & 68 & -179 & 79 & 6 \\
89 & 14039 & 4507 & 23 & -127 & 25 & 14 \\
90 & 14033 & 4482 & 19 & -156 & 30 & 21 \\
91 & 14028 & 4489 & 19 & -153 & 21 & 27 \\
92 & 14022 & 4482 & 19 & -151 & 23 & 32 \\
93 & 14016 & 4486 & 21 & -169 & 25 & 29 \\
94 & 14011 & 4493 & 19 & -138 & 28 & 27 \\
95 & 14005 & 4504 & 21 & -155 & 27 & 22 \\
96 & 13999 & 4489 & 28 & -156 & 31 & 15 \\
97 & 13994 & 4489 & 22 & -208 & 28 & 14 \\
98 & 13988 & 4504 & 35 & -180 & 59 & 9 \\
99 & 13983 & 4486 & 80 & -130 & 74 & 7 \\
100 & 13977 & 4464 & 41 & -74 & 37 & 9 \\
101 & 13971 & 4425 & 100 & -144 & 79 & 5 \\
102 & 13966 & 4529 & 69 & -157 & 44 & 7 \\
103 & 13960 & 4475 & 53 & -66 & 68 & 7 \\
104 & 13955 & 4511 & 45 & -136 & 52 & 6 \\
105 & 13949 & 4493 & 43 & -100 & 53 & 6 \\
\hline \hline
\end{tabular}

${ }^{*}$ Reference position is RA $(J 2000)=101959.9, \mathrm{DEC}(\mathrm{J} 2000)=081334.0$

Anita M. S. Richards, Dr. P. Thomasson, Dr. T. Muxlow, and all staff of MERLIN for their kind help.

\section{References}

Diamond, P.J., et al. 1999, ApJ, 511, 178

Haschick, A.D., Bann, W.A. \& Peng, E.W. 1994, ApJ, 437, L35

Lonsdale, C.J., et al. 1998, ApJ, 493, L13

Mirabel, I.F. \& Sanders, D.B. 1987, ApJ, 322, 688

Watson, W.D. \& Wallin, B.K. 1994, ApJ, 432, L35

Yates, J.A., et al. 2000, MNRAS 317, 28

Yu Zhi-yao, 1996, Astrophys. \& Space Sci., 241, 305 